\title{
CONSTANT MEAN CURVATURE DISCS WITH BOUNDED AREA
}

\author{
RAFAEL LÓPEZ AND SEBASTIÁN MONTIEL
}

(Communicated by Peter Li)

\begin{abstract}
It has been long conjectured that the two spherical caps are then only discs in the Euclidean three-space $\mathbb{R}^{3}$ with non-zero constant mean curvature spanning a round circle. In this work, we prove that it is true when the area of such a disc is less than or equal to that of the big spherical cap.
\end{abstract}

\section{INTRODUCTION AND STATEMENT OF RESULTS}

We shall consider the problem of classifying all the compact surfaces in the Euclidean space $\mathbb{R}^{3}$ with non-zero constant mean curvature $H$ spanning a radius one circle. Heinz $[\mathrm{H}]$ found that a necessary condition for existence in this situation is $|H| \leq 1$. So, we shall suppose $0<|H| \leq 1$. The only known examples are the following: the spherical caps with radius $1 /|H|$ (two non-congruent if $|H|<1$ with areas $A_{+}, A_{-}$respectively) which are the only umbilical ones and some (non-embedded) surfaces of genus bigger than two whose existence was shown by Kapouleas in [K]. This lack of examples and the analogy with the boundaryless case provides evidence supporting the two following conjectures:

Conjecture 1. An immersed disc with non-zero constant mean curvature spanning a circle must be a spherical cap.

Conjecture 2. An embedded compact surface with non-zero constant mean curvature spanning a circle must be a spherical cap.

Of course, these are the boundary case versions of the celebrated theorems by Hopf and Alexandrov respectively. Partial answers to the second question can be seen in [E-B-M-R] and [B-E]. In this paper we solve affirmatively Conjecture 1 provided that the area of our immersed disc is less than or equal to the area of the big spherical cap spanning the given circle. In fact, we prove

Theorem. Let $\phi: D \rightarrow \mathbb{R}^{3}$ be an immersion of the two-dimensional disc in the Euclidean space with constant mean curvature $H, 0<|H| \leq 1$, such that $\phi(\partial D)$

Received by the editors July 29, 1993.

1991 Mathematics Subject Classification. Primary 53A10; Secondary 53C42.

Key words and phrases. Constant mean curvature, disc, isoperimetric inequality.

The authors were partially supported by DGICYT Grant No. PB91-0731. 
is a radius one circle. Then the area $A$ of $\phi(D)$ satisfies

$$
A \geq A_{-}=\frac{2 \pi}{H^{2}}\left(1-\sqrt{1-H^{2}}\right)
$$

where $A_{-}$is the area of the small spherical cap of radius $\frac{1}{|H|}$ spanning $\phi(\partial(D))$. Moreover, the equality holds if and only if $\phi$ is umbilical and so $\phi(D)$ is this small spherical cap.

By combining this theorem and an isoperimetric inequality due to Barbosa and Do Carmo ([B-C]) we obtain

Corollary. If the area of an immersed disc in $\mathbb{R}^{3}$ with constant mean curvature $0<|H| \leq 1$ spanning a radius one circle satisfies

$$
A \leq A_{+}=\frac{2 \pi}{H^{2}}\left(1+\sqrt{1-H^{2}}\right),
$$

then the immersion is umbilical and its image is either the big or the small spherical cap.

\section{Proof}

Consider an immersion $\phi: D \rightarrow \mathbb{R}^{3}$ from the two-dimensional disc into the Euclidean space having non-zero constant mean curvature $H$ and such that

$$
\phi(\partial D)=\left\{p \in \mathbb{R}^{3} ;|p|^{2}=1,\langle p, a\rangle=0\right\}
$$

for a certain unit vector $a \in \mathbb{R}^{3}$, that is, $\phi(\partial D)$ is a radius one circle (so $|H| \leq 1$ by the Heinz result). By endowing $D$ with the Riemannian metric $d s^{2}$ induced by $\phi$ from the Euclidean one of $\mathbb{R}^{3}$ we get a compact simply connected Riemannian surface. The following isoperimetric inequality due to Barbosa and Do Carmo is valid (see [B-C] and [B-Z]):

$$
L^{2}-2 A\left(2 \pi-\int_{D}(K-k)^{+} d A\right)+k A^{2} \geq 0
$$

where $A$ is the area of $D, L$ the length of $\partial D, K$ the Gaussian curvature function, $d A$ the canonical measure associated to the metric $d s^{2}$, and $k$ an arbitrary real number.

In our case we know that $K \leq H^{2}$ with equality holding only at the umbilical points and, on the other hand, $L=2 \pi$ because $\phi(\partial D)$ is a radius one circle of $\mathbb{R}^{3}$. So, taking $k=H^{2}$ in the inequality above

$$
0 \leq H^{2} A^{2}-4 \pi A+4 \pi^{2},
$$

that is, either

or

$$
A \leq A_{-}=\frac{2 \pi}{H^{2}}\left(1-\sqrt{1-H^{2}}\right)
$$

$$
A \geq A_{+}=\frac{2 \pi}{H^{2}}\left(1+\sqrt{1-H^{2}}\right) .
$$

If some of these two inequalities become an equality, then we have $K=H^{2}$ and so our immersion would be umbilical.

Now we need to use a certain flux formula which appears in [K-K-S] given for the embedded case, but that is true also for the immersed case. For completeness, we shall give a proof of this formula. 
Lemma. Let $M$ be a compact surface with boundary $\partial M$ and $\phi: M \rightarrow \mathbb{R}^{3}$ be an immersion with constant mean curvature $H$. We denote by $\alpha: \partial M \rightarrow \mathbb{R}^{3}$ the restriction of $\phi$ and by $N: M \rightarrow \mathbb{R}^{3}$ the Gauss map of $\phi$. Then

$$
H \int_{\partial M} \alpha \wedge \alpha^{\prime}=-\int_{\partial M} N \wedge \alpha^{\prime} .
$$

Proof. We define an $\mathbb{R}^{3}$-valued one form $\omega$ on the surface $M$ by

$$
\omega_{p}(v)=(H \phi+N)_{p} \wedge(d \phi)_{p}(v), \quad p \in M, v \in T_{p} M .
$$

As $H$ is a constant, one easily sees that $d \omega=0$, that is, $\omega$ is closed. From Stokes's theorem, we have

$$
\int_{\partial M} \omega=0
$$

that is,

$$
\int_{\partial M}(H \alpha+N) \wedge \alpha^{\prime}=0 .
$$

Now, we can continue with the proof of the theorem. We shall denote by $k_{g}$ the geodesic curvature of $\partial D$ in $\left(D, d s^{2}\right)$. Since $\phi(\partial D)$ is a circle of radius one, $k_{g}^{2}=1-k_{n}^{2}$, where $k_{n}$ is the normal curvature of $\phi(D)$ in the direction of $\alpha^{\prime}(s)$. We choose the parametrization of $\phi(\partial D)$ to get $\alpha \wedge \alpha^{\prime}=a$, where $a=(0,0,1)$. Then

$$
\begin{aligned}
k_{n} & =-\left\langle N^{\prime}, \alpha^{\prime}\right\rangle=\left\langle N, \alpha^{\prime \prime}\right\rangle \\
& =-\langle N, \alpha\rangle=\left\langle\alpha^{\prime} \wedge N, a\right\rangle .
\end{aligned}
$$

The lemma gives us

$$
\int_{\partial D} k_{n}=2 \pi H
$$

So, by using the Cauchy-Schwarz inequality,

$$
\begin{aligned}
4 \pi^{2} H^{2} & \leq 2 \pi \int_{\partial D} k_{n}^{2}=4 \pi^{2}-2 \pi \int_{\partial D} k_{g}^{2} \\
& \leq 4 \pi^{2}-\left(\int_{\partial D} k_{g}\right)^{2} .
\end{aligned}
$$

Then we get

$$
\left|\int_{\partial D} k_{g}\right| \leq 2 \pi \sqrt{1-H^{2}}
$$

From the last inequality and using the Gauss-Bonnet theorem, we obtain

$$
\begin{aligned}
2 \pi & =\int_{D} K+\int_{\partial D} k_{g} \leq A H^{2}+\int_{\partial D} k_{g} \\
& \leq A H^{2}+2 \pi \sqrt{1-H^{2}},
\end{aligned}
$$

and the proof is finished

With respect to the proof of the corollary, we only remark that if $A \leq A_{+}$, then, from the theorem and the previous isoperimetric inequality, we get $A=$ $A_{-}$or $A=A_{+}$, i.e., $\phi$ is umbilical and its image is a (small or big respectively) spherical cap. 


\section{REFERENCES}

[B-C] J. L. Barbosa and M. do Carmo, A proof of a general isoperimetric inequality for surfaces, Math. Z. 173 (1980), 13-28.

[B-E] F. Brito and R. S. Earp, Geometric configurations of constant mean curvature surfaces with planar boundary, Ann. Acad. Brasil. Ciênc. 63 (1991), 5-19.

[B-Z] Y. Burago and V. Zalgaller, Geometric inequalities, Springer-Verlag, New York, 1988.

[E-B-M-R] R. Earp, F. Brito, W. H. Meeks III, and H. Rosenberg, Structure theorems for constant mean curvature surfaces bounded by a planar curve, Indiana Univ. Math. J. 40 (1991), 333-343.

[H] H. Heinz, On the nonexistence of a surface of constant mean curvature with finite area and prescribed rectifiable boundary, Arch. Rational Mach. Anal. 35 (1969), 249-252.

[K] N. Kapouleas, Compact constant mean curvature surfaces in Euclidean three-space, J. Differential Geom. 33 (1991), 683-715.

[K-K-S] N. Korevaar, R. Kusner, and B. Solomon, The structure of complete embedded surfaces with constant mean curvature, J. Differential Geom. 30 (1989), 465-503.

Departamento de Geometria y Topologfa, Universidad de Granada, Facultad de Ciencias, 18071 Granada, Spain

E-mail address: rlopez@ugr.es 